\title{
Lossless image compression by LMS adaptive filter banks
}

\author{
Ruşen Öktem ${ }^{\mathrm{a}}$, A. Enis Çetin ${ }^{\mathrm{b}, *}$, Omer N. Gerek ${ }^{\mathrm{c}}$, Levent Öktemª, \\ Karen Egiazarian $^{\mathrm{a}}$ \\ ${ }^{a}$ Signal Processing Laboratory, Tampere University of Technology, P.O. Box 553, FIN 33101 Tampere, Finland \\ ${ }^{\mathrm{b}}$ Department of Electrical and Electronics Engineering, Bilkent University, Bilkent, TR 06533 Ankara, Turkey \\ 'Anadolu University, Eskisehir, Turkey
}

Received 7 August 2000

\begin{abstract}
A lossless image compression algorithm based on adaptive subband decomposition is proposed. The subband decomposition is achieved by a two-channel LMS adaptive filter bank. The resulting coefficients are lossy coded first, and then the residual error between the lossy and error-free coefficients is compressed. The locations and the magnitudes of the nonzero coefficients are encoded separately by an hierarchical enumerative coding method. The locations of the nonzero coefficients in children bands are predicted from those in the parent band. The proposed compression algorithm, on the average, provides higher compression ratios than the state-of-the-art methods. (C) 2001 Elsevier Science B.V. All rights reserved.
\end{abstract}

Keywords: Adaptive subband decomposition; Lossless image compression; LMS algorithm

\section{Introduction}

Lossless compression of images is required in many practical applications including medical and space imaging for archiving or transmission. Early lossless image coders, e.g. JPEG lossless mode, are based on DPCM. Wu and Memon improved DPCM schemes by using adaptive prediction and context modeling in CALIC [13]. In [9], an efficient progressive lossless compression is achieved by introducing $\mathrm{S}+\mathrm{P}$ transform, a subband decomposition scheme, and an embedded entropy

\footnotetext{
* Corresponding author. Tel.: + 90-312-266-4126; fax: + 90312-266-4126.

E-mail address: cetin@ee.bilkent.edu.tr (A.E. Çetin).
}

coding. In [2,3], perfect reconstruction filter banks (PRFB) employing adaptive LMS filters are introduced for subband decomposition, and they are used for lossy image compression in [3,6].

In this paper, we use the adaptive PRFB structure in a lossless image compression algorithm and propose to code the subband coefficients by the method developed in [7] which exploits the multiresolution structure of the subband decomposition/wavelet transform.

\section{LMS adaptive prediction filter banks}

The concept of the adaptive filterbanks is introduced in $[1,4,12]$. Classical adaptive prediction concepts are combined with the PRFB in [2,3] 


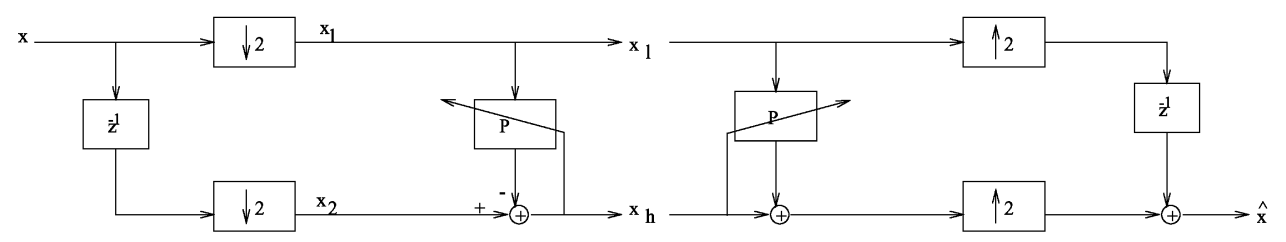

Fig. 1. Analysis and synthesis stages of the 2-channel adaptive filter bank structure.

where the key idea is to decorrelate the polyphase components of the multichannel structure by using an adaptive predictor $P$ (see Fig. 1). The adaptation of the predictor coefficients is carried out by the least mean square (LMS) algorithm, and this helps to cope with the unstationary behavior of the input data.

In Fig. $1, x_{1}(n)$ is the downsampled version of the original signal, $x(n)$, thus it consists of the even samples of $x(n)$. Similarly, the signal $x_{2}(n)$ consists of the odd samples. An LMS-based FIR predictor of $x_{2}(n)$ from $x_{1}(n)$ can be expressed as

$\hat{x}_{2}(n)=\boldsymbol{w}(n) \boldsymbol{x}_{1}^{\mathrm{T}}(n)$,

where $\boldsymbol{x}_{1}(n)=\left[x_{1}(n-N), \ldots, x_{1}(n+M)\right]^{\mathrm{T}}$ is the observation vector, and $\boldsymbol{w}(n)$ is the vector of predictor coefficients which is adapted by the equation

$\boldsymbol{w}(n+1)=\boldsymbol{w}(n)+\mu \frac{\boldsymbol{x}_{1}(n) e(n)}{\left\|\boldsymbol{x}_{1}(n)\right\|^{2}}$,

where the error signal $e(n)$ is given by

$e(n)=x_{2}(n)-\hat{x}_{2}(n)$.

The subband decomposition structure shown in Fig. 1 compacts the most of the energy in the lowest resolution band, and the resulting subsignals are expected to be decorrelated. A weakness of the structure shown in Fig. 1 is that the subsignal $x_{1}(n)$ may suffer from aliasing due to downsampling. Aliasing affects the quality of prediction especially when further decompositions over $x_{1}(n)$ are carried out. In order to eliminate this problem an antialiasing filtering stage is introduced in [3], where $x(n)$ is lowpass filtered by a halfband filter of the form

$H_{1}(z)=\frac{1}{2}\left[1+z^{-1} A\left(z^{2}\right)\right]$.
With the use of the so-called "noble identity" [11], the lowpass filtering operation can be carried out after downsampling as shown in Fig. 2, and the subsignal $x_{2}(n)$ is predicted using $x_{1}(n)$ which is a smoothed version of $x_{1}(n)$.

The above adaptive PRFB structures are extended to two dimensions in a separable manner.

\section{Application to lossless image compression}

The decomposition structure in Fig. 2 is used for lossless image compression as follows: input image is decomposed into multiresolution bands by consecutive row-wise and column-wise operations. In order to obtain integer-valued coefficients, both the predictor output and the low-resolution coefficients $\boldsymbol{x}_{1}$, are rounded to the nearest integer at each stage. This rounding operation makes the perfect reconstruction of $\left\{\boldsymbol{x}_{1}\right\}$ impossible. It is experimentally observed that transmitting the error between $\left\{\boldsymbol{x}_{1}\right\}$ and $\left\{\hat{\boldsymbol{x}}_{1}\right\}$ is generally costly. To overcome this problem, we apply quantization to $\left\{\boldsymbol{x}_{1}\right\}$ and transmit the quantized coefficients first. The decoder reconstructs $\left\{\hat{\boldsymbol{x}}_{1}\right\}$ by using $Q\left(\left\{\boldsymbol{x}_{1}\right\}\right)$, where $Q$ denotes quantization. Then, we transmit the residual error between $\left\{\boldsymbol{x}_{1}\right\}$ and $\left\{\hat{\boldsymbol{x}}_{1}\right\}$. Both the subband decomposition coefficients and the residual error exhibit a multiresolution structure such that locations of the nonzero coefficients in a band can be predicted from those in the coarser band. Hence, both are coded by using the method in [7], which was developed for lossy coding of the wavelet transform coefficients. The method of [7] is an improved version of the scheme in [10]. Its two most important features are scanning the coefficients of a band 


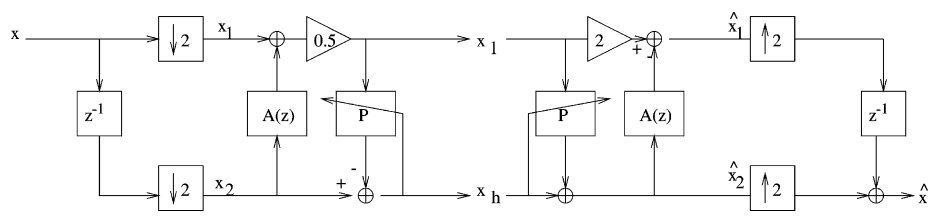

Fig. 2. Adaptive filter bank structure with an antialiasing filter.

by magnitude preference, and employment of hierarchical enumerative coding [5] instead of arithmetic coding.

\section{Experimental results}

We used six $512 \times 512$ images and two $256 \times 256$ images, House and Cameraman, for testing our algorithm. The prediction neighborhood of a pixel is depicted in Fig. 3. We check the variances of left diagonal neighborhood $\{d 1, d 2, d 3\}$, right diagonal neighborhood $\{d 4, d 5, d 6\}$, and horizontal neighborhood $\{h 1, h 2, h 3, h 4\}$ of the pixel to be predicted (shaded in Fig. 3), and use the one with minimum variance if its variance does not exceed a threshold. Otherwise, we use the neighborhood $\{d 2, n 1, d 5$, $h 3, h 4, d 6\}$. The bit-rate results in Table 1 show that the proposed algorithm, on the average, achieves better performance than lossless JPEG [8], S + P [9], and CALIC [13] codecs. In this study, $\mathrm{H}(3)=\left(1+z^{-1}\right) / z$ in Fig. 2.

\section{Conclusions}

A lossless image compression algorithm using multiresolution decomposition by LMS adaptive PRFB is proposed. The algorithm primarily transmits lossy coefficients and then transmits the residual error. The locations of the nonzero coefficients in children bands are predicted from those in the parent band through morphological dilation. Both the location and the magnitude information are entropy coded by hierarchical enumerative coding. The proposed compression algorithm, on the average, achieves a better bit-rate efficiency than that of the state-of-the-art lossless codecs, while proposing progressive transmission.

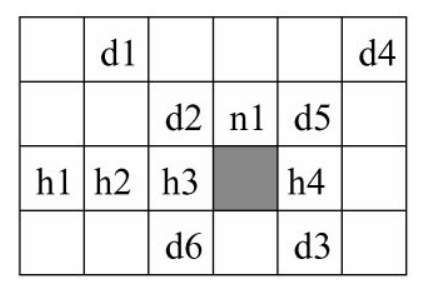

Fig. 3. Prediction neighborhood in columnwise processing.

Table 1

Bit-rate results for different lossless compression methods

\begin{tabular}{lllll}
\hline Images & JPEG & $\mathrm{S}+\mathrm{P}$ & CALIC & $\begin{array}{l}\text { Proposed } \\
\text { method }\end{array}$ \\
\hline Lenna & 4.71 & 4.18 & 4.12 & 3.96 \\
Mandrill & 6.38 & 5.93 & 5.88 & 5.52 \\
Harbour & 5.23 & 4.73 & 4.44 & 4.64 \\
Doex & 5.59 & 4.18 & 3.79 & 3.81 \\
House & 4.95 & 4.22 & 4.00 & 3.57 \\
Cameraman & 5.51 & 4.48 & 4.19 & 3.83 \\
Barbara & 5.58 & 4.69 & 4.63 & 4.59 \\
Goldhill & 5.65 & 4.75 & 4.63 & 4.51 \\
\hline
\end{tabular}

\section{References}

[1] O. Egger, W. Li, M. Kunt, High compression image coding using an adaptive morphological subband decomposition, Proc. IEEE 83 (2) (February 1995) 272-287.

[2] Ö.N. Gerek, A.E. Çetin, Linear/nonlinear adaptive polyphase subband decomposition structures for image compression, ICASSP 98, Seattle, WA, Vol. 3, 12-15 May 1998, pp. 1345-1348.

[3] Ö.N. Gerek, A.E. Çetin, Adaptive polyphase subband decomposition structures for image compression, IEEE Trans. Image Process. 9 (2000) 1649-1660.

[4] F.J. Hampson, J.C. Pesquet, A nonlinear subband decomposition structure with perfect reconstruction, IEEE International Conference on Image Proceeding (1996). 
[5] L. Öktem, J. Astola, Hierarchical enumerative coding of locally stationary binary data, Electr. Lett. 35 (23) (1999) 2003-2005.

[6] R. Öktem, K. Egiazarian, E. Çetin, Subband decomposition based image compression algorithms with nonlinear adaptive filter banks, NSIP 99, Antalya, Turkey, Vol. 2, June 1999, pp. 766-769.

[7] L. Öktem, R. Öktem, K. Egiazarian, J. Astola, Efficient encoding of the significance maps in wavelet based image compression, ISCAS 2000, Geneva, Switzerland, 2000, to appear.

[8] W.B. Pennebaker, J.L. Mitchell, JPEG: Still Image Compression Standard, Van Nostrand Reinhold, New York, 1993.
[9] A. Said, W.A. Pearlman, An image multiresolution representation for lossless and lossy image compression, IEEE Trans. Image Process. 5 (September 1996) 1303-1310.

[10] S.D. Servetto, K. Ramchandran, M.T. Orchard, Image coding based on a morphological representation of wavelet data, IEEE Trans. Image Process. 8 (9) (1999) 1161-1174.

[11] G. Strang, Wavelets and Filter Banks, Wellesley - Cambridge Press, Wellesley, MA, 1996.

[12] W. Sweldens, The lifting scheme: A new philosophy in biorthogonal wavelet constructions, Proc. of SPIE 2569 (September 1995) 68-79.

[13] X. Wu, N. Memon, Context-based, adaptive, lossless image coding, IEEE Trans. Commun. 45 (4) (1997) 437-444. 